ORIGINAL ARTICLE

\title{
Characterising the individual performance responses to mild illness in international swimmers
}

\author{
D B Pyne, W G Hopkins, A M Batterham, M Gleeson, P A Fricker
}

Br J Sports Med 2005;39:752-756. doi: 10.1136/bjsm.2004.017475

See end of article for authors' affiliations

....................

Correspondence to: Dr D B Pyne, Australian Institute of Sport, PO Box 176, Belconnen,

Canberra, ACT 2616

Australia; david.pyne@

ausport.gov.au

Accepted

27 February 2005

\begin{abstract}
Objectives: To determine individual differences in the impact of illness on the change in performance of swimmers in international competitions.

Methods: Subjects were members of the Australian swimming team (33 male and 39 female, aged 15-27 years). Swimmers provided a weekly seven day recall of symptoms of illness during final six weeks of preparations for international competition over a three year period. Swimmers were categorised as either ill (one or more episodes of illness) or healthy. The measure of performances was the international point score. Mean changes in points score were calculated for healthy and ill swimmers between a national championship and an international competition ( 16 weeks later). Likelihoods of substantial effects of illness on an individual's true change in performance (beneficial/trivial/harmful) were estimated from means and standard deviations, assuming a smallest substantial change of 6 points.

Results: Illness was reported before international performances by $38 \%$ of female and $35 \%$ of male swimmers. For female swimmers the change in performance was - 3.7 (21.5) points (mean (SD)) with illness and -2.6 (19.0) points when healthy; for male swimmers the changes were $-1.4(17.5)$ points with illness and 5.6 (13.2) points when healthy. The likelihoods that illness had a substantial beneficial/trivial/ harmful effect on performance of an individual swimmer were $32 \% / 31 \% / 37 \%$ for female and $17 \% / 31 \%$ / $52 \%$ for male participants (90\% confidence limits $\sim \pm 10 \%$ to $20 \%$ ).

Conclusions: Although mild illness had only a trivial mean effect on female swimmers and a small harmful mean effect on male swimmers, there were substantial chances of benefit and harm for individuals.
\end{abstract}

Ilness is experienced by almost $90 \%$ of athletes during training for competitions at some point during a given calendar year. ${ }^{1}$ Although various epidemiological aspects of illness (for example, frequency, incidence, prevalence, duration, severity, and type) in athletic populations have been described in the sports medicine literature, ${ }^{2-4}$ the impact of illness on competitive performance remains uncertain. A clearer understanding of the quantitative impact of illness on athletic performance would assist the sports medicine practitioner, coach, and athlete in assessing the need for prophylaxis before competition.

In an earlier report by our research group, illness in Australian national team swimmers was associated with a trend for impaired international competitive performance. ${ }^{5}$ The effect of illness on performance in that study was unclear, partly because we interpreted the relation between illness and performance from the single perspective of statistical significance. There may or may not have been a good chance that the true effect was substantial, and our interpretation of the outcome and recommendation to athletes and coaches might therefore be different if we had considered the practical significance of illness on performance. A more clinically oriented approach holds that the practical significance of changes and differences in performance measures of elite athletes should be considered when evaluating the true impact of illness or other intervention on performance. ${ }^{6}$ Consideration of the practical significance overcomes concerns that results not statistically significant are often falsely discarded, yet the magnitude of the effect may well be important in athletic events where a small change in performance could mean the difference between winning and losing. ${ }^{78}$ In simple terms the practical outcome of an intervention on athletic performance can be divided into three discrete categories: a worthwhile improvement, a substantial impairment, or a trivial change. Another limitation of the earlier study was a small sample size. To give more certainty about the effects of illness on performance, we have now studied clinical records and swimming performance over three consecutive years.

We also sought to examine the issue of individual responses to illness and to determine whether this effect is of similar magnitude to the mean effect. Although interpreting the individual effects of a treatment or intervention has been described in various clinical settings, ${ }^{9}{ }^{10}$ no previous study has addressed the issue of individual responses in the context of competitive sporting performance. The purpose of this study was to examine the impact of illness on competitive performance by quantifying the likelihood of a practically significant change in performance, in terms of both the mean effect in the population of elite swimmers and for an individual swimmer presenting with illness.

\section{METHODS \\ Subjects}

The sample was 33 male and 39 female members of the Australian swimming team (age range 15 to 29 years). In all, 133 performances (69 male and 64 female performances) were analysed over three consecutive calendar years. The international competitions during this period included the Olympic Games, Pan Pacific Championships, and the Commonwealth Games. Subjects gave written informed consent for medical surveillance as part of their national team medical support programme administered by the national governing body of the sport, Australian Swimming Inc.

Abbreviations: IPS, international point score 
Table 1 International points score at baseline (a national championship) for swimmers who were subsequently healthy or ill before the international competition

\begin{tabular}{lllll}
\hline & Status & & & \\
\cline { 2 - 5 } & Healthy & Sample size & III & $\begin{array}{l}\text { Sample } \\
\text { size }\end{array}$ \\
\hline Female & $954(31)$ & 40 & $942(27)$ & 24 \\
Male & $972(22)$ & 45 & $973(26)$ & 24 \\
\hline
\end{tabular}

Data are mean (SD) of the international point score (IPS).

\section{Performance}

Competition performance was assessed by the official international point score (IPS) system used by the international swimming federation (Fédération Internationale de Natation Amateur - FINA). The mean time of the eight fastest swims in history in each event is ascribed the value of 1000 points, with individual performances rated against this reference value. This system allows comparison of a given competitive performance by a male or female athlete in any of the sanctioned events (that is, freestyle, butterfly, backstroke, breast stroke, and individual medley). The scoring system is used by leading coaches and swimmers worldwide, and is accessible at http://www.swimnews.com/ranking/ ips.jhtml. During the taper period before the competitions we did not keep detailed training records for individual swimmers, but the competition performances were generally of a high standard for the Australian swimming team. In general, the course of the training programme followed the traditional decrease in training volume, reduced number of sessions, and development of swimming speed.

\section{Monitoring illness}

Symptoms of illness were monitored over a six week period including the taper phase and the major international competition in each calendar year. Swimmers free of symptoms during the study period were categorised as healthy, while those reporting illness ( subject to the inclusion criteria) were categorised as ill. Symptoms were self reported on a seven day recall basis and confirmed by interview with the team physician and study investigators. The criteria for inclusion of a discrete episode of illness were that symptoms were reported for two or more consecutive days, or required modification of the training or competition programme on any given day. No severe episodes of illness were recorded and in every case the swimmer recovered sufficiently to compete in their selected event at the international competition. All swimmers were already preselected for their competitive events limiting the possibility of underreporting of illness for fear of non-selection in the international competition.

Symptoms were primarily mild to moderate self limiting respiratory illness in origin, although a small number of gastrointestinal and viral skin conditions were also reported.

Table 2 Effect of being healthy or ill before an international competition on mean change in international points score (IPS) since the preceding national championship

\begin{tabular}{|c|c|c|c|}
\hline & \multicolumn{2}{|l|}{ Status } & \multirow{2}{*}{$\begin{array}{l}\text { III-healthy } \\
(90 \% \mathrm{Cl})\end{array}$} \\
\hline & Healthy & III & \\
\hline $\begin{array}{l}\text { Female swimmers } \\
\text { Male swimmers }\end{array}$ & $\begin{array}{l}-2.6(19.0) \\
5.6(13.2)\end{array}$ & $\begin{array}{l}-3.7(21.5) \\
-1.4(17.5)\end{array}$ & $\begin{array}{l}-1.1( \pm 8.9) \\
-7.0( \pm 6.9)\end{array}$ \\
\hline
\end{tabular}

Values are mean (SD) with $90 \%$ confidence intervals (Cl).
The swimmers were asked to report any of the following symptoms: respiratory (sneezing, stuffy nose, runny nose, sore throat); lower respiratory (cough, sputum, chest congestion); generalised viral illness (aching joints, fever, and chills); gastrointestinal (nausea, vomiting, and diarrhoea); eye irritation (itchy, red, water, allergy); and skin conditions (spots, rashes, measles, skin infections). We cannot exclude the possibility that some of the upper respiratory symptoms may have been caused by allergic rhinitis but this is unlikely given that known polluted areas were not visited.

\section{Statistical analysis}

Competitive performance in the national and international swimming meets is presented as mean (between-subject SD). The degree of uncertainty in the estimate of the true (population) mean change in performance was expressed as $90 \%$ confidence intervals $(90 \% \mathrm{CI})$. The unequal variances $t$ statistic was used to analyse differences in the mean change in IPS points for competitive performances in swimmers with illness compared with their healthy counterparts. To determine whether the better swimmers had the same response to illness as the poorer swimmers we conducted an analysis of covariance for the effect of IPS in the nationals on change in IPS.

The uncertainty in the effect of illness on competitive swimming performance was calculated using the percent chance or likelihood that the true value of the effect was substantial (greater than the smallest practically important effect) and interpreted qualitatively. Thresholds for assigning the qualitative terms to chances of substantial effects were: $<1 \%$, almost certainly not; $<5 \%$, very unlikely; $<25 \%$, unlikely or probably not; $<50 \%$, possibly not; $>50 \%$, possibly;
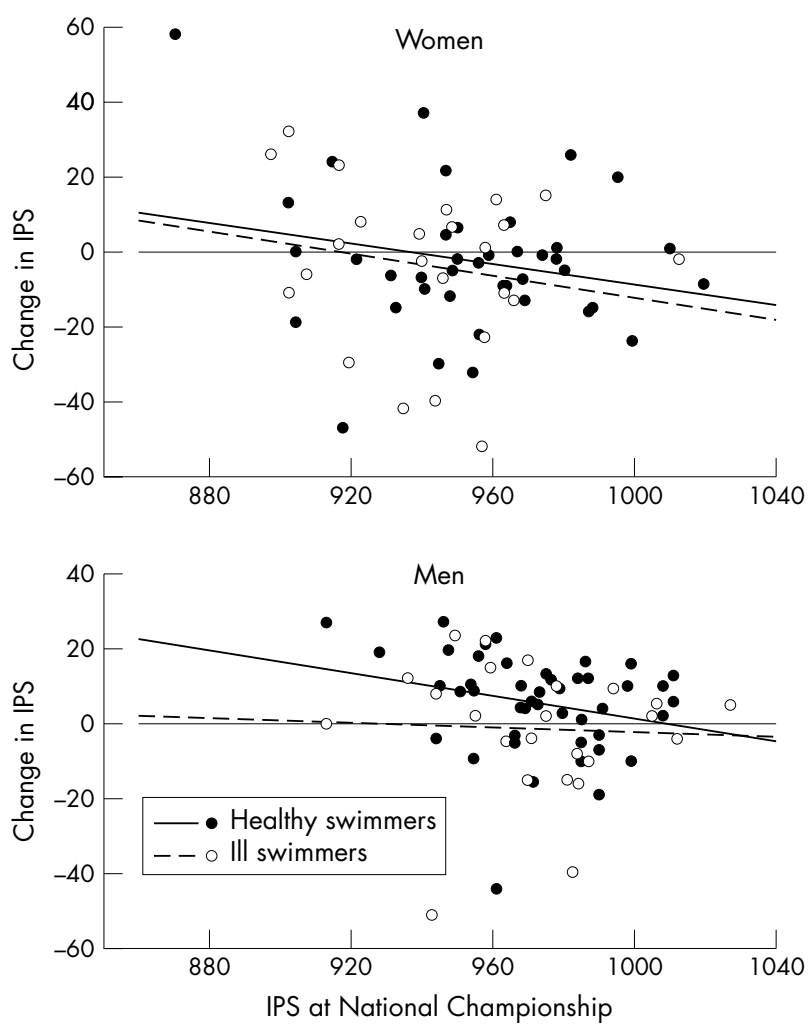

Figure 1 Change in international points score (IPS) between a national and international competition in relation to performance at the national competition for female and male swimmers who were healthy or ill before the international competition. 
Table 3 Chances that an individual swimmer would show a beneficial, trivial, or harmful change in competitive performance after reporting symptoms of illness

\begin{tabular}{llll}
\hline & Beneficial & Trivial & Harmful \\
\hline Female swimmers & $32(20$ to 52$)$ & $31(14$ to 35$)$ & $37(21$ to 59$)$ \\
Male swimmers & $17(10$ to 34$)$ & 30 (16 to 43) & 53 (33 to 69) \\
\hline Values are mean (90\% confidence interval). &
\end{tabular}

$>75 \%$, likely or probable; $>95 \%$, very likely; and $>99 \%$ almost certain. ${ }^{11}$

Estimation of the magnitude of the smallest worthwhile change or effect in terms of international swimming performance was based on the methods outlined previously. ${ }^{6}$ In brief, the smallest worthwhile change in performance for an elite athlete is approximately 0.5 of the within-athlete standard deviation in performance between competitions. ${ }^{6}{ }^{12} 13$ The within-athlete variation for international swimmers is approximately $0.8 \%$ of performance time. ${ }^{14}$ To determine the magnitude of this value for the current study, we examined the IPS listings for the 2003 calendar year for all male and female freestyle events from the $50 \mathrm{~m}$ to the $1500 \mathrm{~m}$. A difference in performance time equating to $0.4 \%$ (that is, $0.5 \times 0.8 \%$ ) at the internationally competitive level of 950 IPS was six points and we therefore assigned this value as the smallest worthwhile change.

To estimate the effect of illness on the performance of individual swimmers, we first estimated a standard deviation representing individual responses (the square root of the difference in the variances in the change scores in the illness and healthy groups). ${ }^{15}$ We combined this standard deviation with the standard error of the mean effect of illness to derive the sampling distribution for a swimmer's true response to illness. Degrees of freedom for the distribution were computed via the Satterthwaite approximation. ${ }^{16}$ Chances for beneficial, trivial, and harmful changes were estimated by assuming a smallest worthwhile change in the IPS of 6 . Confidence limits for the estimates were derived by bootstrapping. The two primary outcomes of interest were: the likelihood that illness would reduce the chance of a given swimmer substantially improving their performance, and the likelihood that illness would increase the chance of a substantial decline in performance.

\section{RESULTS}

\section{Characteristics of the sample}

Over the three year study period illness was reported before $35 \%$ of international performances in male swimmers and before $38 \%$ of performances in female swimmers. For male and female swimmers combined there were 18 performances associated with illness (44\% of total performances) in the first year of the study, 17 (35\%) in the second, and $13(30 \%)$ in the final year. Table 1 shows IPS at baseline (the national championships) for swimmers who were subsequently healthy or ill before the major international competition.

\section{Mean response to illness}

The mean effects of illness on the change in IPS between the national and international competitions are shown in table 2. Illness was associated with a trivial impairment of mean performance in the female swimmers and a small impairment in the male swimmers. These uncertainties can be expressed as chances of the true mean effect being beneficial/ trivial/harmful; the chances were $0 \% / 40 \% / 60 \%$ for the male swimmers and $9 \% / 73 \% / 18 \%$ for the female swimmers. Thus for male swimmers, illness is almost certainly not beneficial and possibly harmful, whereas for female swimmers illness is unlikely to be beneficial or harmful.

\section{Individual response to illness}

The IPS change scores for individuals plotted against their baseline (national) scores are shown in fig 1. All groups showed trends towards an inverse relation between baseline score and change score. The difference between the trends for the healthy and ill male swimmers implies that illness had a greater effect on slower swimmers; the healthy-ill difference in the IPS change score at a baseline score of 960 was 8.5 (90\% CI, \pm 8.5$)$, whereas the difference for the fastest swimmers (baseline of 1030) was negligible.

The standard deviation of the change scores was greater for the ill swimmers than for the healthy swimmers (table 2), indicating that there were individual responses in the effects of illness on performance. Expressed as standard deviations, the individual responses were 12 points (90\% CI, -5 to 17 ) and 10 points ( -13 to 19) for the male swimmers and female swimmers, respectively. These individual responses, combined with the uncertainty in the mean effect of illness, provided estimates of chances that being ill (relative to being healthy) would have a beneficial/trivial/harmful effect on performance of an individual (table 3). It appears that an individual male swimmer reporting illness had a threefold greater chance of his competitive performance being harmed than improved.

\section{DISCUSSION}

Given the limitations of relying solely on statistical significance to interpret the often subtle factors influencing international level sporting performance, ${ }^{78^{17} 18}$ we calculated the likelihood that the true change in performance is greater than a predetermined smallest important or worthwhile change. ${ }^{17}$ In female swimmers, the mean effects of illness on performance were trivial. The male swimmers with illness had a mean reduction of 7.0 points (equating to a time differential of $\sim 0.5 \mathrm{~s}$ over a $200 \mathrm{~m}$ race) in the IPS rating compared with their healthy team mates, a magnitude that is slightly greater than the smallest worthwhile change of 6.0 points. The pertinent question is how likely is it that the true change for the population of international swimmers is greater than -6.0 points, given $90 \%$ confidence limits of -0.1 to -13.9 . The chances of a true reduction in performance with illness were $60 \%$, or odds of approximately 2 to 1 . Hence for male swimmers, mild illness between competitions "possibly" results in a substantial decline in performance. Based on this study the advice to the coach or swimmer would be that "the odds are only 2 to 1 or $\sim 60 \%$ that illness experienced during the taper period will lead to a substantial decrease in performance." Trivial to small effects of illness are not surprising, considering that the athletes were well enough to compete. On the basis of these results, clinicians can reassure athletes that symptoms of mild illness during the final few weeks of a preparation do not necessarily lead to a substantial decline in subsequent performance.

Notwithstanding these trivial to small mean effects of illness on performance, the analysis of individual responses indicates that the mean effect does not characterise the effect in individual swimmers adequately. Indeed, the standard deviation representing individual responses, defined as the typical variation in the response to illness from individual to individual, was substantial in both male and female swimmers, though a larger sample size is needed to reduce its uncertainty. Importantly, this standard deviation is free from within-subject measurement error, as this source of error may have an appreciable bearing on the magnitude of the individual response to the predictor variable ${ }^{18}$-in this case the presence of illness. The individual responses analysis 
What is already known on this topic

The potential for illness to disrupt training and competition schedules of athletes is well understood. Although various epidemiological aspects of illness and changes in immune function have been described, the impact on performance remains uncertain. A clearer understanding of the quantitative impact of illness would assist team personnel in the management of health and training issues.

revealed that on average 32 of every 100 female swimmers and 17 of every 100 male swimmers may actually benefit from illness. This contrasts with the lower likelihoods for benefit based on the mean response. However, the substantial risk of harm for the individual underscores the responsibility of coaches, athletes, and team personnel for the implementation of practical and self management strategies. ${ }^{19} 20$ These strategies include management of physical training loads, psychological stress, environmental conditions, dietary and self hygiene practices, and clinical considerations including treatment and prophylactic therapeutic intervention. ${ }^{19}{ }^{21}$

The apparently beneficial effect of mild illness for some individuals may be the result of a period of enforced rest during the taper period. Given the evidence of individual responses to illness in this investigation, future studies should focus on identifying the subject characteristics and other factors that predict them. This process would involve using these factors as covariates in the statistical analysis of predictor and outcome variables. Potential factors accounting for variation in the individual response to illness are likely to include the following: the severity, duration, and timing of illness; physical training loads; the presence of psychological stress; nutritional status; immune status; medical history and pre-existing medical conditions; and the degree of pathogen exposure. $^{319}$ The data showing a distribution of changes in performance from impaired to improved are consistent with the findings of an earlier study showing a range from $-1.1 \%$ to $+6.0 \%$ during the taper in swimmers before the 2000 Olympic Games. ${ }^{22}$

One characteristic that did account for individual responses to a small extent was the ability of the athlete, as represented by the IPS in the national competitions. The analysis of covariance for the effect of IPS in the nationals on the change in IPS between competitions suggested that the effect of illness tended to be negligible for top male swimmers, with little effect of illness on the chances of female swimmers swimming better or worse. However, the difference between the trends for the healthy and ill male swimmers implies that illness had a greater effect on slower swimmers. It is unclear why this response was observed in the male but not the female swimmers. One possible explanation is that male swimmers suffering symptoms of illness may have had a tendency to give up, whereas their female counterparts maintained their effort irrespective of the underlying health status.

This study is the first to quantify individual responses to illness on sporting performance. Our method of expressing individual responses as chances of benefit and harm is closely related to the recent practice in clinical research of estimating the number needed to treat before one patient benefits (NNB) and the number needed to treat before one patient is harmed $(\mathrm{NNH})^{10}$ : chances of benefit and harm are simply $100 / \mathrm{NNB}$ and 100/NNH, respectively. We have found it easier to understand the implications for individuals when individual responses are expressed as chances of benefit and harm rather than as numbers needed to treat for benefit and harm.
What this study adds

This study is the first to quantify the effects of mild illness on the competitive performance of elite swimmers. The results showed trivial to small mean effects, but the chances of harm for individuals were substantial. Practical strategies for limiting the risk of illness are needed on a case by case basis.

We also advise strongly against combining beneficial and harmful outcomes into a single number needed to treat, as proposed by Guyatt and coworkers, ${ }^{9}$ because it is important for everyone to understand the likelihood of positive and negative consequences of a condition or a treatment on health or performance.

In summary, the analysis of the mean responses suggested that mild illness had a trivial effect on the competitive performances of female swimmers, and a substantial though small harmful effect in male swimmers. Although these mean effects were trivial to small, the chances of harm for individuals were substantial, and team personnel should therefore be aware of the need to implement practical strategies for limiting the risk of illness. Given the relatively small sample size and the degree of uncertainty in the estimates provided in this study, further investigations are required to clarify the likelihood of mild, moderate, and severe illness impairing athletic performance. Studies examining the performance of athletes should consider both the mean effects and the individual responses to a treatment or intervention in terms of the likelihood of a beneficial, trivial, or harmful outcome. The findings of the present investigation should assist clinicians in framing their advice to athletes and coaches on the implications of illness on performance.

\section{ACKNOWLEDGEMENTS}

We wish to acknowledge the cooperation of the swimmers, coaches, and support staff of the Australian Swimming Team.

\section{Authors' affiliations}

D B Pyne, P A Fricker, Australian Institute of Sport, Canberra, Australia W G Hopkins, Auckland University of Technology, Auckland, New Zealand

A M Batterham, University of Bath, Bath, Somerset, UK

M Gleeson, Hunter Area Pathology Service, Singleton, NSW, Australia

Competing interests: none declared

\section{REFERENCES}

1 Fricker PA, Gleeson M, Flanagan A, et al. A clinical snapshot: do elite swimmers experience more upper respiratory illness than non-athletes? J Clin Exerc Physiol 2000;2:155-8.

2 Shephard RJ, Shek PN. Exercise training and immune function. In: HoffmanGoetz L, eds. Exercise and immune function. Boca Raton: CRC Press, 1996:93-119.

3 Shephard RJ, Shek PN. Exercise, immunity and susceptibility to infection. A Jshaped relationship? Phys Sportsmed 1999;27:47-71.

4 Brenner IKM, Shek PN, Shephard RJ. Infection in athletes. Sports Med 1994;17:86-107.

5 Pyne D, McDonald W, Gleeson M, et al. Mucosal immunity, respiratory illness, and competitive performance in elite swimmers. Med Sci Sports Exerc $2001 ; 33: 348-53$.

6 Hopkins WG, Hawley JA, Burke LM. Design and analysis of research on sport performance enhancement. Med Sci Sports Exerc 1999;31:472-85.

7 Andersen MB, Stoove MA. The sanctity of $p<0.05$ obfuscates the good stuff: a comment on Kerr and Goss, J App/ Sport Psychol 1998;10:168-73.

8 Stoove MA, Andersen MB. What are we looking at, and how big is it? Phys Ther Sport 2003;4:93-7.

9 Guyatt GH, Juniper EF, Walter SD, et al. Interpreting treatment effects in randomised trials. BMJ 1998;316:690-3.

10 Dalton GW, Keating JL. Number needed to treat: a statistic relevant for physical therapists. Phys Ther 2000;80:1214-19.

11 Liow DK, Hopkins WG. Velocity specificity of weight training for kayak sprint performance. Med Sci Sports Exerc 2003;35:1232-37. 
12 Hopkins WG Hewson DJ. Variability of competitive performance of distance runners. Med Sci Sports Exerc 2001;33:1588-92.

13 Stewart AM, Hopkins WG. Consistency of swimming performance within and between competitions. Med Sci Sports Exerc 2000;32:997-1001.

14 Pyne DB, Trewin CB, Hopkins WG. Progression and variability of competitive performance of Olympic swimmers. J Sports Sci 2004;22:613-20.

15 Hopkins WG. A spreadsheet for analysis of straightforward controlled trials Sportscience 7, 2003; sportsci.org/jour/03/wghtrials. htm: accessed 4 July, 2004.

16 Satterthwaite FW. An approximate distribution of estimate of variance components. Biometrics Bull 1946;2:110-14.

17 Hopkins WG. Statistical vs clinical or practical significance. Sportscience 6, 2002; sportsci.org/jour/0201/Statistical_vs_clinical. ppt: accessed 4 July, 2004
18 Shephard RJ, Rankinen T, Bouchard C. Test-retest errors and the apparent heterogeneity of training response. Eur J Appl Physiol 2004;91:199-203.

19 Pyne DB, Gleeson M, McDonald WA, et al. Training strategies to maintain immunocompetence in athletes. Int $J$ Sports Med 2000;21:S51-60.

20 Gleeson $M$. The scientific basis of practical strategies to maintain immunocompetence in elite athletes. Exerc Immunol Rev 2000;6:75-101.

21 Cox A, Gleeson M, Pyne D, et al. Valtrex therapy for Epstein-Barr virus reactivation and upper respiratory symptoms in elite runners. Med Sci Sports Exerc 2004:36:1104-10.

22 Mujika I, Padilla S, Pyne D. Swimming performance changes during the final 3 weeks of training leading to the Sydney 2000 Olympic Games. Int J Sports Med 2002;23:582-7.

\section{Clinical Evidence-Call for contributors}

Clinical Evidence is a regularly updated evidence-based journal available worldwide both as a paper version and on the internet. Clinical Evidence needs to recruit a number of new contributors. Contributors are healthcare professionals or epidemiologists with experience in evidence-based medicine and the ability to write in a concise and structured way.

Areas for which we are currently seeking authors:

- Child health: nocturnal enuresis

- Eye disorders: bacterial conjunctivitis

- Male health: prostate cancer (metastatic)

- Women's health: pre-menstrual syndrome; pyelonephritis in non-pregnant women However, we are always looking for others, so do not let this list discourage you.

Being a contributor involves:

- Selecting from a validated, screened search (performed by in-house Information Specialists) epidemiologically sound studies for inclusion.

- Documenting your decisions about which studies to include on an inclusion and exclusion form, which we keep on file.

- Writing the text to a highly structured template (about 1500-3000 words), using evidence from the final studies chosen, within 8-10 weeks of receiving the literature search.

- Working with Clinical Evidence editors to ensure that the final text meets epidemiological and style standards.

- Updating the text every six months using any new, sound evidence that becomes available. The Clinical Evidence in-house team will conduct the searches for contributors; your task is simply to filter out high quality studies and incorporate them in the existing text.

- To expand the topic to include a new question about once every 12-18 months.

If you would like to become a contributor for Clinical Evidence or require more information about what this involves please send your contact details and a copy of your CV, clearly stating the clinical area you are interested in, to Klara Brunnhuber (kbrunnhuber@ bmigroup.com).

\section{Call for peer reviewers}

Clinical Evidence also needs to recruit a number of new peer reviewers specifically with an interest in the clinical areas stated above, and also others related to general practice. Peer reviewers are healthcare professionals or epidemiologists with experience in evidence-based medicine. As a peer reviewer you would be asked for your views on the clinical relevance, validity, and accessibility of specific topics within the journal, and their usefulness to the intended audience (international generalists and healthcare professionals, possibly with limited statistical knowledge). Topics are usually 1500-3000 words in length and we would ask you to review between $2-5$ topics per year. The peer review process takes place throughout the year, and our turnaround time for each review is ideally 10-14 days.

If you are interested in becoming a peer reviewer for Clinical Evidence, please complete the peer review questionnaire at www.clinicalevidence.com or contact Klara Brunnhuber (kbrunnhuber@bmigroup.com). 